\title{
Circadian Cycle and Its Influence on Parameters of Aerobic Training
}

\author{
Ramon Cruz ${ }^{1}$, Francisco de Assis Manoel ${ }^{2}$, Bruno Pereira Melo², Sandro Fernandes da Silva ${ }^{3, *}$ \\ ${ }^{1}$ Faculdade de Educação Física e Desporto, Universidade Federal de Juiz de Fora, Juiz de Fora, Brazil \\ ${ }^{2}$ Departamento de Educação Física, Universidade Estadual de Maringá, Maringá, Brazil \\ ${ }^{3}$ Departamento de Educação Física, Universidade Federal de Lavras, Lavras, Brazil \\ *Corresponding author: sandrofs@gmail.com
}

Received January 29, 2014; Revised March 05, 2014; Accepted March 09, 2014

\begin{abstract}
Objectives: This study aims to evaluate the Influence of two distinct Periods of the day on maximal aerobic power in young Athletes mountain bike. Volunteers are the mountain bike Athletes, $37.83 \pm 8.87$ years, and underwent two progressive maximal exercise test on a cycle Ergometer at two times of day. Gauging the behavior glucose and heart rate. The verification of normality of data was performed by the Shapiro-Wilk test for analysis of variables was Adopted $t$ test for paired samples. Statistical we Adopted an evidence for $\mathrm{p}<0.05$. Results: Were the glucose threshold results not statistically significant. However trends indicating were there a better outcome in the evening. The maximal workload, maximal heart rate and glycemic load threshold. Conclusions: The results show trends on performance and circadian rhythm, and yet, the routine or team chosen for sensivity training appears to the circadian cycle.
\end{abstract}

Keywords: circadian cycle, training and cycling

Cite This Article: Ramon Cruz, Francisco de Assis Manoel, Bruno Pereira Melo, and Sandro Fernandes da Silva, "Circadian Cycle and Its Influence on Parameters of Aerobic Training." American Journal of Sports Science and Medicine, vol. 2, no. 2 (2014): 65-69. doi: 10.12691/ajssm-2-2-2.

\section{Introduction}

The performance variables physiological and psychological suffers interference of biological rhythms, which are cyclic changes that are regularly repeated in a given time interval generated in periods of 24 hours [1]. Some studies had been made identifying the influence of circadian cycle in determining variables on performance, as cardiovascular function [8], metabolic [9] and respiratory [2].

Physiological control of the circadian cycle is determined by changes in frequency environmental, and the light / dark cycle is one of the main synchronizers. Another way for the body to control their circadian mechanisms is necessary external and internal stimuli, acting as guides. These two pieces of information are transmitted to a biological oscillator, located in the suprachiasmatic nucleus of the hypothalamus, which acts as an integrator [3].

The interferon Consistency of the circadian cycle in human performance seems to follow the rhythm of body temperature [4,5]. Body temperature is often used as a marker rhythm, ease of measurement and its strong endogenous component. The minimum body temperature curve occurs during sleep, around 4:00 [7]. From this point, it starts increasing which increases upon awakening. After noon, there is a slight decrease, a phenomenon called post-lunch dip, then rise again, which leads to the occurrence of higher values at about 18:00 [9]. Similar to body temperature, heart function has also changes according to time of day, consistently lower at evening, independent of load, with a difference between day and night for 5-10 bpm.

The HR max has been studied by different researchers $[1,6]$. In the literature we apply the cycle ergometer exercise at various times, such as: 2:00, 6:00, 10:00, 2:00pm, 6:00pm and 10:00pm and has been found that significant circadian variation in the $\mathrm{Fc}_{\text {max }}$ [10]. Moreover, also the use of cycling were their used the time, 4:00, 8:00, 12:00, 3:00pm, 6:00pm, 9:00pm and 24:00 h [11], and in another study that found no significant differences between the different periods [5], used four times (8:00, 12:00, 4:00pm and 8:00pm) [5].

For best response to training, it is necessary to control some markers of performance. An important marker of human performance, $\mathrm{VO}_{2 \mathrm{MAX}}$ shows a circadian cycle of the interference [6] as well as other markers performance aerobic and anaerobic most [1,6] such as lactate, and the critical velocity (CV) [12].

The anaerobic threshold (AT) is a parameter widely used for exercise prescription and research in exercise physiology [13]. The AT can be determined by the behavior of blood lactate, which is a body's metabolic response to stress and that means a gradual transition between the energy systems [11]. Yet another method has been highlighted in order to identify the AT, which is the blood glucose response during progressive exercise [5]. But the blood glucose response has yet been the focus of many studies seeking its new relationship with other 
existing parameters, especially the blood lactate response to exercise [5]. The literature is still scarce in checking the influence of circadian cycle in glycemic threshold (GT).

This study presents an interesting practical application for the prescription of cyclists training, checking the best period for the implementation of training and performance in different periods.Thus this work aims to evaluate the influence of circadian cycle in GT and physiological variables determined in a progressive maximal test.

\section{Methodology}

\subsection{Sample}

There were 8 subjects in the study used adult, $37.83 \pm$ 8.87 years, $174.7 \mathrm{~cm} \pm 8.01,71.52 \pm 11.87 \mathrm{~kg}, 20.26 \pm$ $2.56 \%$ fat men, athletes mountain bike, with activities other than sport. All were competitive period of training, participating in regional and state exams. All subjects were pre-informed about the risks of the project and they all signed the Informed Consent approved by the ethics committee of the University of Itaúna number of protocol 002/09, following the Brazilian regulations for research on human beings, which are in accordance with the norms proposed by the treaty of Helsinki

\subsection{Procedures}

Subjects came to the laboratory three times, first for two days before the start of testing, for explanation of the study, signing the WIC and evaluation of body composition. The second visit occurred 48 hours after the 1 st, where the subjects performed a maximal exercise test on a cycle ergometer, the ú last visit occurred at an interval of 96 hours of a first visit. Assessments were made on two moments in the morning between 7:00 and $10: 00$ hours and at evening from $7: 00 \mathrm{pm}$ to $10: 00 \mathrm{pm}$ hours. The subjects randomly performed stress tests subject to a maximum 48-hour interval between assessments for the full recovery of muscle glycogen stores and liver.

\subsection{Protocol to the Maximal Exercise Test}

A progressive test was used which consisted of an initial load of $15 \mathrm{~W}$ then by increments of 30 watts every 2 minutes until exhaustion, with the frequency being maintained between 80 and 90 revolutions per minute (rpm). The test was interrupted with the exhaustion of the individual, with the impossibility of sustaining the pace of pedaling or pre-established with the detection of any event which could cause possible damage to the volunteer. At the end of each stage was performed to collect blood samples for analysis of glucose in a glucose meter Acutrend Roche ${ }^{\circledR}$.

\subsection{Collection of Blood Glucose}

The collection of blood glucose was performed in three stages in this study: 30 minutes before the test immediately before the test and each stage.

At such times, $25 \mathrm{~mL}$ were collected blood from the right ear lobe of individuals and then this drop was placed on a strip for analysis of specific Acutrend Roche ${ }^{\circledR}$ Plus, which measures the concentration of glucose in plasma by strips reagentes ${ }^{\circledR}$ by Roche metering method of reflection in order to obtain the blood glucose concentration in these moments.

\subsection{Statistical Analysis}

For the analysis of all variables obtained was used descriptive statistics to deter nation of mean and standard deviation. The verification of normality of data was performed by the Shapiro-Wilk. To identify the statistical differences between the predictor variables, Gluc Fc, $F_{\text {max }}$ load and max load Gluc, we adopted the $t$ test for paired samples. For statistical evidence we adopted a p < 0.05 .

\section{Results}

The first graphic shows the average in all subjects reached the GT, these values are distinct threshold presented in Figure 2 and Figure 3, because here we consider the average of the entire group, not the average of each load. With these results we find relative values for the threshold glycemic load, as follows: $73.78 \%$ of the maximum load for the morning and $73.05 \%$ for the evening time. The charges glycemic threshold showed no significant difference $(\mathrm{p}>0,05)$.

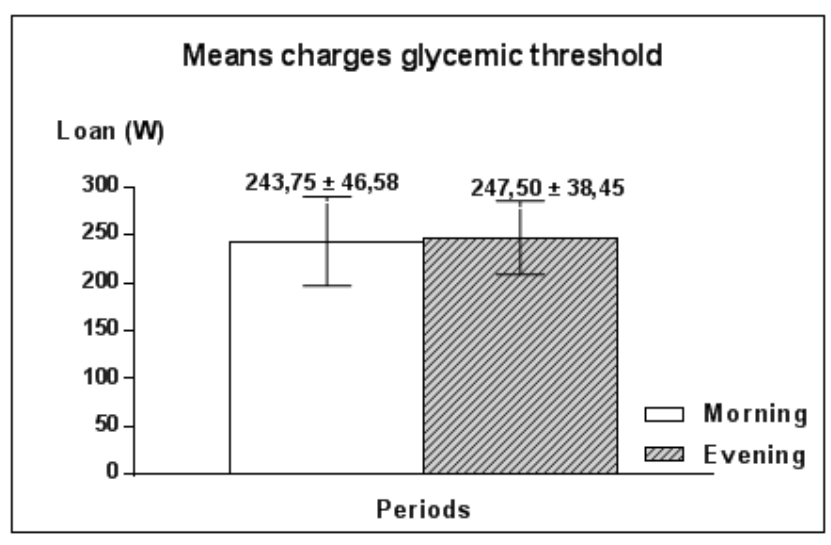

Figure 1. Mean and SD results in loads when the volunteers reached the glycemic threshold

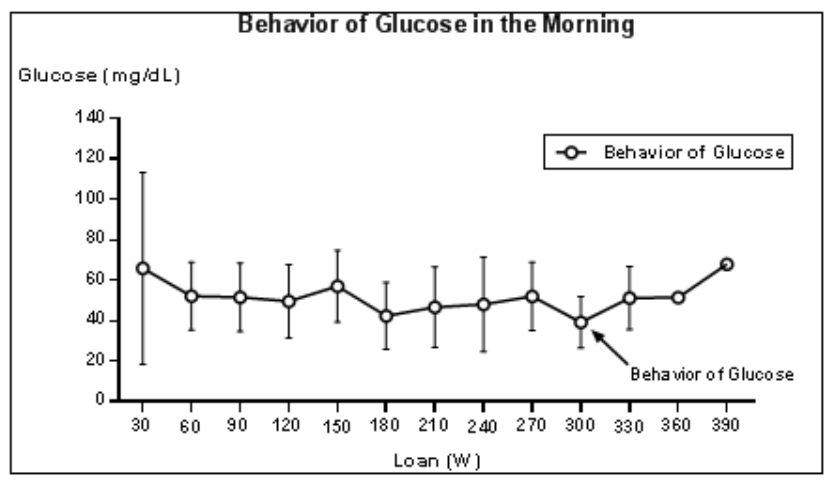

Figure 2. Behavior of glucose in the morning, with mean and SD of the volunteers on each charge, and identification of the glycemic threshold. The curve was smoothed by the software Excel

Figure 2 and Figure 3 show the average values at each load in 2 periods of study evaluation, and have identified the glycemic thresholds at every turn. These graphs are paradoxical when compared to a chart, they show different charges for the GT [13], however, remember that the 
glycemic curves presented show the values of the volunteers at each stage, which does not necessarily reflect the the load at which individuals reached their GT. The curves (lines) have been smoothed for better visualization of the graph tool available in Excel ${ }^{\circledR}$ software.

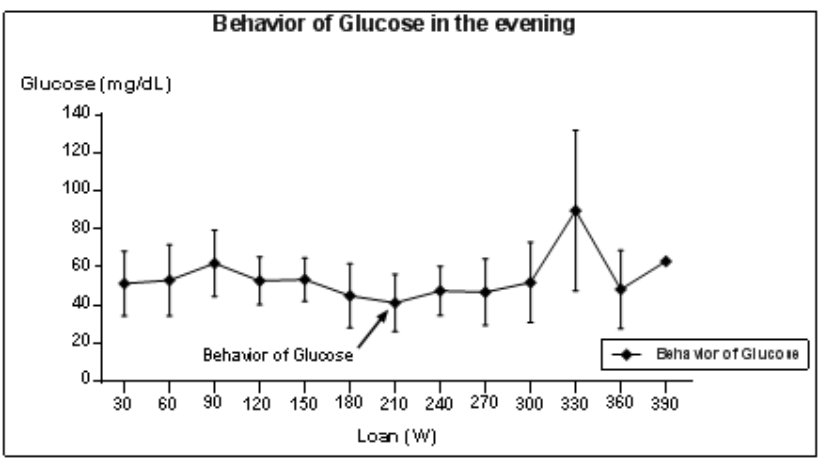

Figure 3. Behavior of glycemic during the evening, with mean and SD of the volunteers on each charge, and identification of the glycemic threshold. The curve was smoothed by the software Excel

Figure 4 shows the mean values of the loads that were achieved in both periods. Nah it was a statistically significant difference; however there is a behavior that indicates best performance at evening. The maximum load showed no significant difference $(\mathrm{p}>0,05)$.

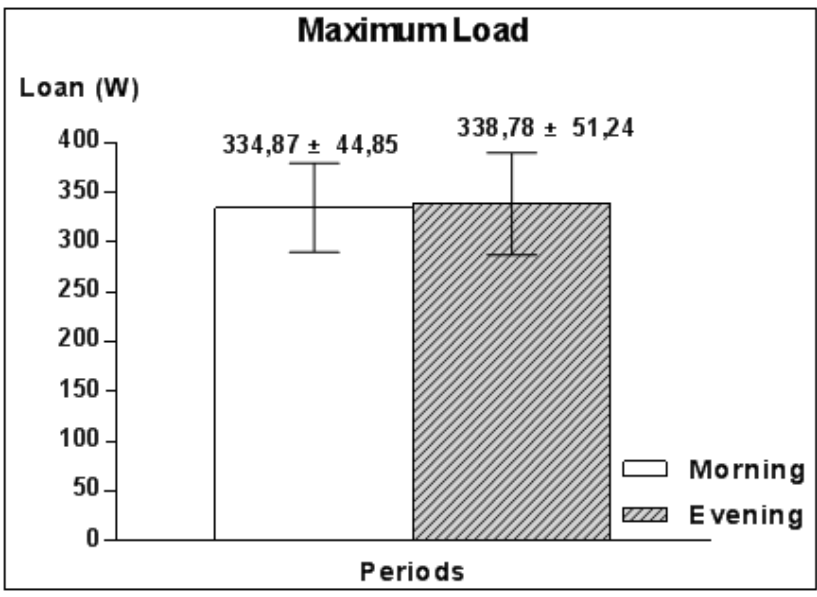

Figure 4. Mean and SD results observed in the analysis of the maximum load obtained in the tests in both periods

Table 1 contains the values of body temperature, observed in both periods. The evening the average temperature was higher, which corroborates with the literature $^{2}$. The mean body temperature showed no significant difference $(\mathrm{p}>0,05)$.

Table 1. Mean and SD of the temperature in the times of the test Mean body temperature $\left({ }^{\circ} \mathrm{C}\right)$

\begin{tabular}{cc}
\hline Morning & $35,55 \pm 0,62$ \\
Evening & $35,9 \pm 0,4$
\end{tabular}

The Figure 5 and Figure 6 bring the curves of the values $\mathrm{m} s$ edition of HR and blood glucose levels by goodwill of the progressive test. The heart rate values are displayed on each of the graphic marker. Being that at night it was lower in the first 7 stages, and higher in the last six.

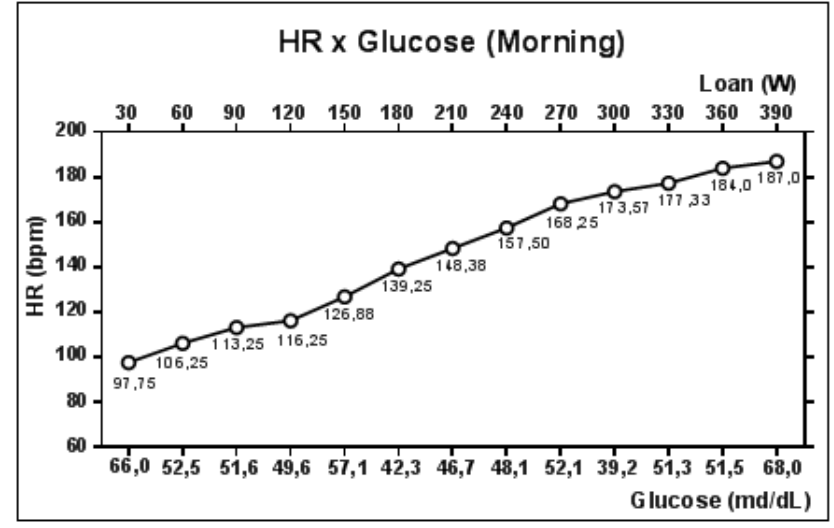

Figure 5. Describes the mean values of $\mathrm{HR}$ in each stage with the average values of glycemic at each stage in the morning

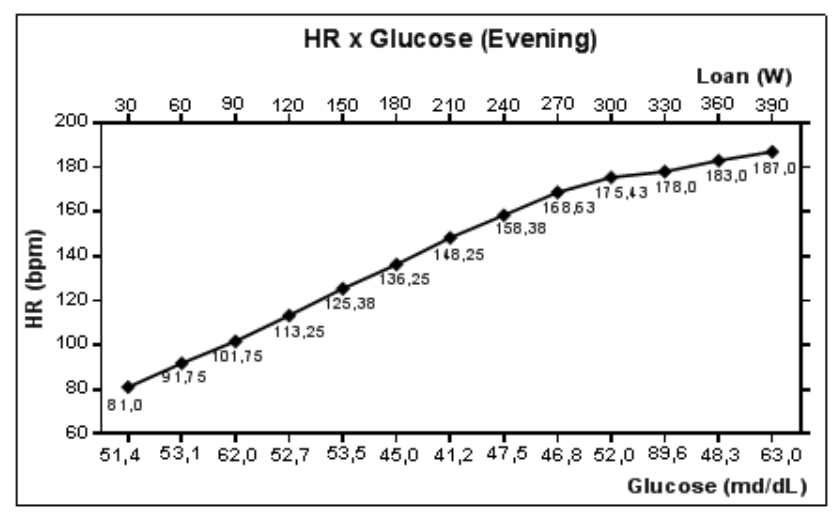

Figure 6. This graph depicts the mean values of HR in each stage with the average values of glycemic at each stage during the evening.

Table 2 shows that the average maximum HR attained in both tests was higher at evening. During this period the volunteers were great physiological responses to athletic performance (HR, Temperature, GT). The HR máx (morning and evening) showed no significant difference $(\mathrm{p}>0,05)$.

Table 2. Mean and SD results observed in the analysis of maximum HR obtained in the tests in two periods

$$
\text { HR máx (Mornng and Evening) }
$$

\begin{tabular}{cc}
\hline Morning & $179,75 \pm 5,17 \mathrm{bpm}$ \\
Evening & $181,25 \pm 8,71 \mathrm{bpm}$ \\
\hline
\end{tabular}

\section{Discussion}

The aim of this study was to investigate the interference of the circadian cycle in GT and physiological variables determined in this metabolic transition point, the current literature there are no studies that evaluate this important predictor of performance, what about the proposed work extremely attractive.

The results of this study showed no statistically significant difference, similar to other studies with this population and research problem [4,6,14], however these variables showed an average trend, which indicates better performance on tests used at evening. In a previous study by checking the variable speed, showed the same trend of results on the behavior of physical performance on the circadian cycle [12], which we believe that the habits of the volunteers are influential in the final results of the tests. 
The literature suggests that the best moments of peak performance are related to body temperature [15] with a worse performance in the morning and a better outcome in the afternoon / evening [16]. In our study we found a 2\% increase in body temperature, which leads us to think that this increased tendency to perform better during the night can be directly related to the increase in body temperature, in corroborates with other studies which indicate values larger afternoon / evening [16,17,18].

The choice of two periods for evaluations of the study was based on the times when the population in general performs physical activity, not including the time of the afternoon was because we believe that the difference between the hours of the night chosen (from 7:00pm h) and afternoon hours (2:00pm to 6:00pm) would be close, and also the results were not significant between afternoon and evening, observed in a study that preceded this [12].

Maintaining or increasing rates of glucose during exercise can be explained both by the greater needs of glucose by the body as the increased intensity of exercise, promoting greater release of hyperglycemic hormones such as catecholamines, ACTH, glucagon, cortisol and growth hormone $[4,13]$. In our findings there was no interference of the circadian rhythm in blood glucose concentration which led to the charges in Liglic.

The concept arose with Liglic inferences that proposed the identification of anaerobic threshold by comparing the behavior of blood glucose with the behavior of lactate minimum, where similarity was found between these two variables [20]. The GT is identified by the kinetics of glucose concentration [gluc], being found in exercise intensity corresponding to the lowest [gluc] during the test, representing the point in time when the activation hiperglicemic secretion of hormones such as cortisol and that glucagon will maintain the blood glucose concentration during the entire test [20,21]. During exercise maintenance of plasma glucose levels are governed by the liver through feeedback signals, with increased glucose availability [21] these signals are inhibited, which could probably alter the kinetics of glucose at different times of day that could influence the performance of the workload achieved in Liglic.

Studies evaluating the effect of circadian cycle in anaerobic threshold (AT) show contradictory results similar to ours, in which there are differences in AT different times of day [22,23], these studies serve as a parameter in our study since the Liglic presents a metabolic transition, we can infer that the interference of the cycle would continue regardless of the form to determine the Lan

One hypothesis as a possible explanation for the findings in our study is that more than circadian variation, which interferes with physical performance rhythmicity is the time when the subjects performed their usual physical activities, which induce an adaptation of performance and thus would be possible to find differences in the charges assessed in the AT. This study had the limitation that no monitoring of athletes for more hours of the day, as their feed in the moments before the test. Further studies on this topic may further elucidate the influence of time of day on the variables that determine sporting performance.

\section{Conclusion}

The results of this study showed no interference of the circadian cycle in certain variables in the GT. It is noteworthy that there is a tendency for results to be better at evening. What sustains speculated, that the training time of the selected sample is fundamental to happen to a better performance during training and evaluation of physical abilities.

\section{Acknowledgement}

FAPEMIG- Fundação de Amparo à Pesquisa do Estado de Minas Gerais.

\section{References}

[1] Afonso L.S, Dos Santos J.F.B, Lopes J.R, Tambelli R, Santos E.H.R, Back F.A, Menna-barreto L, Lima J.R.P.: Maximal heart rate on treadmill at different times. Rev. Bras. Med. Esporte. 2006, 12 (6): 318-322.

[2] Atkinson G, Jones $H$, Ainslie PN. Circadian variation in the circulatory responses to exercise: relevance to the morning peaks in strokes and cardiac events. Eur J Appl Physiol 108: 15-29, 2010

[3] Atkinson G, Todd C, Reilly T, Waterhouse J. Diurnal variation in cycling performance: influence of warm-up. J Sports Sci. 2005, 23(3): 321-329.

[4] Back FA et al. Non-photic synchronization: the effect of aerobic physical exercise Rev Bras Med Esporte. 2007, 13(2).

[5] Bessot N, Nicolas A, Moussay S, Gauthier A, Sesboüé B, Davenne D. The effect of pedal rate and time of day on the time to exhaustion from high-intensity exercise. Chronobiol Int. 2006, 23(5): 1009-1024.

[6] Cruz R Melo BP Manoel FA Castro PHC da Silva SF. Pacing Strategy and Heart Rate on the Influence of Circadian Rhythms. JEP online. 2013; 16(4):24-31.

[7] Cruz R, Silva SF. Indicators Of Control Parameters Of Training And Circadian Cycle. Rev. Acta Bras. Movimento Hum 2011, 1(1): 33-41.

[8] Drust B, Waterhouse J, Atkinson G, Edwards B, Reilly T. Circadian rhythm in sports performance- An update. Chronobiol Int. 2005, 22: 21-44.

[9] Frank AS et al. Impact of the human circadian system, exercise, and their interaction on cardiovascular function. Proc Natl Acad Sci. 2010, 107(4).

[10] Forsyth J, Reilly T. Circadian rhythms in blood lactate concentration incremental During Ergometer rowing. Eur. J. Appl. Physiol. 2004, 92 (1-2): 69-74.

[11] Hayes LD, Bickerstaff GF, Baker JS. Interactions of cortisol, testosterone, and resistance training: influence of circadian rhythms. Chronobiol Int. 2010, 27(4): 675-705.

[12] Hill DW. Effect of time of day on aerobic power in exhaustive high-intensity exercise. J. Sports Me Phys. Fitness 1996, 36: 155160.

[13] Jones H, George K, Edwards B, Atkinson G. Effects of time of day on post-exercise blood pressure: circadian or sleep-related influences? Chronobiol Int. 2008, 25(6): 987-998.

[14] Racinais S, Connes P, Bishop D, Blonc S, Huel O. Morning versus evening power output and repeated-sprint ability. Chronobiol Int. 2005, 22(6): 1029-1039.

[15] Reilly T, Atkinson G, Gregson W, Drust B, Forsyth J, Edwards B, Waterhouse J. Some chronobiological considerations related to physical exercise. Clin Ter. 2006, 157(3): 249-264.

[16] Sedliak M, Finni T, Cheng S, Kraemer WJ, Häkkinen K. Effect of time-of-day-specific strength training on serum hormone concentrations and isometric strength in men. Chronobiol Int. 2007, 24(6): 1159-1177.

[17] Simões HG, Campbell CSG, Kushnick MR, Nakamura A, Katsanos CS, Baldissera V, Moffatt RJ. Blood glucose threshold and the metabolic responses to incremental exercise tests with and without prior lactic acidosis induction. Eur. J. Appl. Physiol. 2003; 89: 603-11.

[18] Simões HG, Campbell CSG, Kokubum E, Denadai, BS, Baldissera $\mathrm{V}$. Blood glucose response in humans mirror lactate responses for 
individual anaerobic threshold and for lactate minimum in track test. Eur. J. Appl. Physiol. 1999, 80 (1): 34-40.

[19] Souissi N, Driss T, Chamari K, Vandewalle H, Davenne D, Gam A, Fillard JR, Jousselin E. Diurnal variation in wingate test performances: influence of active warm-up. Chronobiol Int. 2010, 27(3): 640-652.

[20] Kline EC, Durstine JL, Davis JM, Moore TA, Devlin TM, Zielinski MR, Youngstedt SD. Circadian variation in swim performance. J. Appl. Physiol. 2007, 102: 641-649.

[21] Souissi N, Souissi M, Souissi H, Chamari K, Tabka Z, Dogui M, Davenne D. Effect of time of day and partial sleep deprivation on short-term, high-power output. Chronobiol Int. 2008, 25(6):10621076.

[22] Waterhouse J, Drust B, Weinert D, Edwards B, Gregson W, Atkinson G, Kao S, Aizawa S, Reilly T. The circadian rhythm of core temperature: origin and some implications for exercise performance. Chronobiol Int. 2005, 22(2): 207-225.

[23] Waterhouse J, Edwards B, Bedford P, Hughes A, Robinson K, Nevill A, Weinert D, Reilly T. Thermoregulation during mild exercise at different circadian times. Chronobiol Int. 2004, 21(2): 253-275. 